Abigail S. Brown, MSN, MBA

Critical Care Transport, Medical Operations,

Emergency Services Institute, Cleveland Clinic
Fredric M. Hustey, MD

Critical Care Transport, Medical Operations,

Center for Emergency Medicine, Emergency

Services Institute, Cleveland Clinic
Anita J. Reddy, MD, MBA

Department of Critical Care Medicine,

Respiratory Institute, Cleveland Clinic

\title{
Interhospital transport of patients with COVID-19: Cleveland Clinic approach
}

\section{Posted June 2, 2020}

\begin{abstract}
Hospital-to-hospital transportation of patients in the COVID-19 era presents unique challenges to ensuring the safety of both patients and health care providers. Crucial factors to address include having adequate supplies of protective equipment and ensuring their appropriate use, defining patient care procedures during transport, and decontamination post-transport. Transport vehicles need to have adequate physical space, an isolated driver compartment, NS HEPA filtration of air. Having a standardized intake process can help identify patients who would benefit from transport to another facility.
\end{abstract}

\section{INTRODUCTION}

Transport of critically ill patients with COVID-19 presents a unique challenge given the risk of transmission for the disease. Most often, patients are transferred from one hospital to another for specialized services or a higher level of care.

Communication and preplanning are key elements to ensure safe transport of these patients and to minimize risk of disease transmission to the transport personnel. ${ }^{1,2}$ Items to consider at intake include necessity of transport, ideal destination facility and unit type, and duration of transport or out-of-hospital time. Subsequent planning includes appropriate use and type of protective equipment, patient care procedures during transport, and decontamination post-transport.

\section{CLEVELAND CLINIC TRANSPORT PLAN}

The Cleveland Clinic critical care transport team (CCT) partnered with the Cleveland Clinic Health System, our vehicle vendors, and the Ohio Depart-

The statements and opinions expressed in COVID-19 Curbside Consults are based on experience and the available literature as of the date posted. While we try to regularly update this content, any offered recommendations cannot be substituted for the clinical judgment of clinicians caring for individual patients.

doi:10.3949/ccjm.87a.ccc045 ment of Health to develop a comprehensive transport management plan for the transport of patients with confirmed or suspected COVID-19 infection. It was finished in about mid-March 2020.

Crucial factors in the plan for the safety of patients and transport personnel include maintaining adequate types and volumes of personal protective equipment (PPE) as well as ensuring that all staff have the appropriate training in regards to PPE management, including donning and doffing. Also needed are patient barriers and device adjuncts based on the patient's clinical situation. Examples include face masks for nonintubated patients, adding HEPA filters at the ventilator exhalation valve or expiratory port of manual resuscitation devices (such as bag-valve-mask devices), and avoiding any disconnects of the ventilator circuit.

Policies and training modules were implemented to address PPE management and PPE recommendations based on risk in transport. For example, when transporting any patients with suspected or confirmed COVID-19, team members are required to wear a gown, gloves, goggles, and surgical mask. For patients at high risk for aerosolization of infectious material (eg, intubated patients), an N95 mask is required. ${ }^{3}$

\section{PATIENT TRANSPORT FACTORS TO CONSIDER}

The environment of the transport vehicle is also an important consideration. This includes having adequate physical space, isolating the driver (or pilot) compartment, providing HEPA filtration of recycled air, ensuring proper PPE, adequate cleaning of the space, and training of all transport personnel. ${ }^{4}$ Additionally, contingency plans should be in place for patient deterioration or medical emergencies during transport, including having additional PPE available for all transport personnel. ${ }^{5}$ Efforts should be made to ensure patient stability prior to transport, with particular emphasis on securing the airway. Having controlled intubation in a closed room will result in 
less aerosolization and, thus, less risk to caregivers versus intubation in a transport vehicle.

Collaboration between the Cleveland Clinic CCT and Cleveland Clinic Institutes and vehicle vendor partners has been crucial to ensuring seamless and safe transport of patients during the COVID-19 pandemic. The CCT provides care in a variety of environments ranging from the emergency department and intensive care unit transfers to prehospital requests with outside emergency management service (EMS) agencies.

\section{ESTABLISHING A PROCESS FOR TRANSFERS}

To standardize the intake process and ensure protection of all health care providers involved with a patient transfer, the CCT clinical coordinator routinely screens all transfer patients with a short COVID19 screening questionnaire at the time of transfer request. As it is often not possible to obtain substantial patient-specific details for EMS or other out-ofhospital transfers prior to on-scene arrival, and due to the urgent and unpredictable environment while on scene, all of these patients are treated as potential COVID-19 cases and managed appropriately.

Additional modifications to the hospital transfer and CCT inter-facility transport request process have been implemented due to the risks of COVID19 transmission. Cleveland Clinic has an external hospital transfer workflow protocol for patients with COVID-19 to help identify which patients may benefit from treatment or therapy otherwise unavailable at the referring facility (including referring capacity issues) (Figure 1).

\section{PATIENT TRIAGE}

Patient transports are generally requested by the referring provider. These requests are then triaged as per standard CCT protocol by an on-duty CCT acute care nurse practitioner (ACNP) or medical director. If discrepancies arise between the referring provider's request and the CCT triage decision, the provider is contacted to discuss the case and come to a decision on the most mode of transport. This adds an extra layer of safety and ensures appropriate and timely transfer of all patients.

During night shifts, the CCT triage may be conducted through the CCT ACNPs who cover the health system's eHospital, which also covers many of the CCHS regional ICUs. As many of our transports occur within the Cleveland Clinic Health System, involvement of eHospital and the CCT ACNPs both leverages additional technology in the triage process and helps to ensure a seamless transition with highly coordinated care as they provide medical direction during transport and real-time updates to the receiving facility.

\section{CONSIDERING TRANSPORT TYPES}

Transportation of COVID-19 patients also affects the consideration of transport mode. Risk of transmission can be best minimized via ground transport. However, there are additional factors to consider for alternate modes of transport including distance, out-of-hospital time, acuity of patients' condition, and time-sensitive diagnoses, as well as the minimum number of CCT personnel required to safely complete each transport. In certain circumstances, air transportation, either rotor or fixed-wing, might be necessary.

Cleveland Clinic medical crews partner with vendors who operate and maintain transport vehicles. Working together with these vendors, guidelines were developed and implemented team-wide to mitigate exposure risk. For ground vehicles, these guidelines recommend having barriers between the patient and driver compartments. Drivers must wear surgical masks. Special accommodations for air transport include considerations for airflow management and pilot protection. For example, fixed-wing transports need to maintain front-to-back airflow without recirculating cabin air. In addition, extra personnel, including family members, are limited on the transport, unless accompanying a pediatric patient.

\section{STOCKING PPE}

Given the various locations of our ground and air transport bases, routinely high transport volumes, and rapidly changing guidelines for PPE use, maintaining adequate and readily accessible supplies of PPE and equipment presents a unique challenge. In addition to standard Cleveland Clinic inpatient PPE guidelines, adjustments were necessary given transportspecific considerations. Initially during the pandemic when COVID-19-related transport volumes were low, stocking all PPE at main campus was preferred for security and tracking purposes. However, as these transport volumes grew and PPE use increased, this process created a potential for delays in returning a vehicle to service when resupply was necessary. Therefore, we developed dedicated PPE bags for all modes of transport that could be managed directly from the local bases (Figure 2).

To preserve PPEs and ensure good stewardship of their use, supply checks and counts from the PPE bag were added to the daily shift checklist and a PPE use log was implemented for tracking purposes. We 


\section{Cleveland Clinic COVID External Hospital Transfer Workflow}

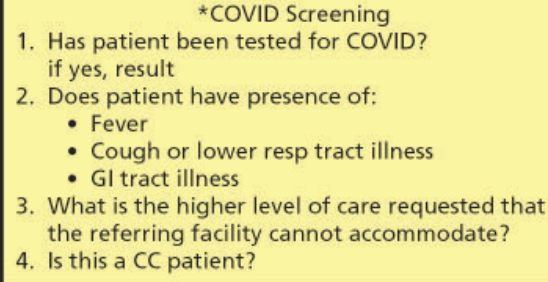

2. Does patient have presence of:

- Fever

- Cough or lower resp tract illness

- Gl tract illness

3. What is the higher level of care requested that the referring facility cannot accommodate? 4. Is this a CC patient?

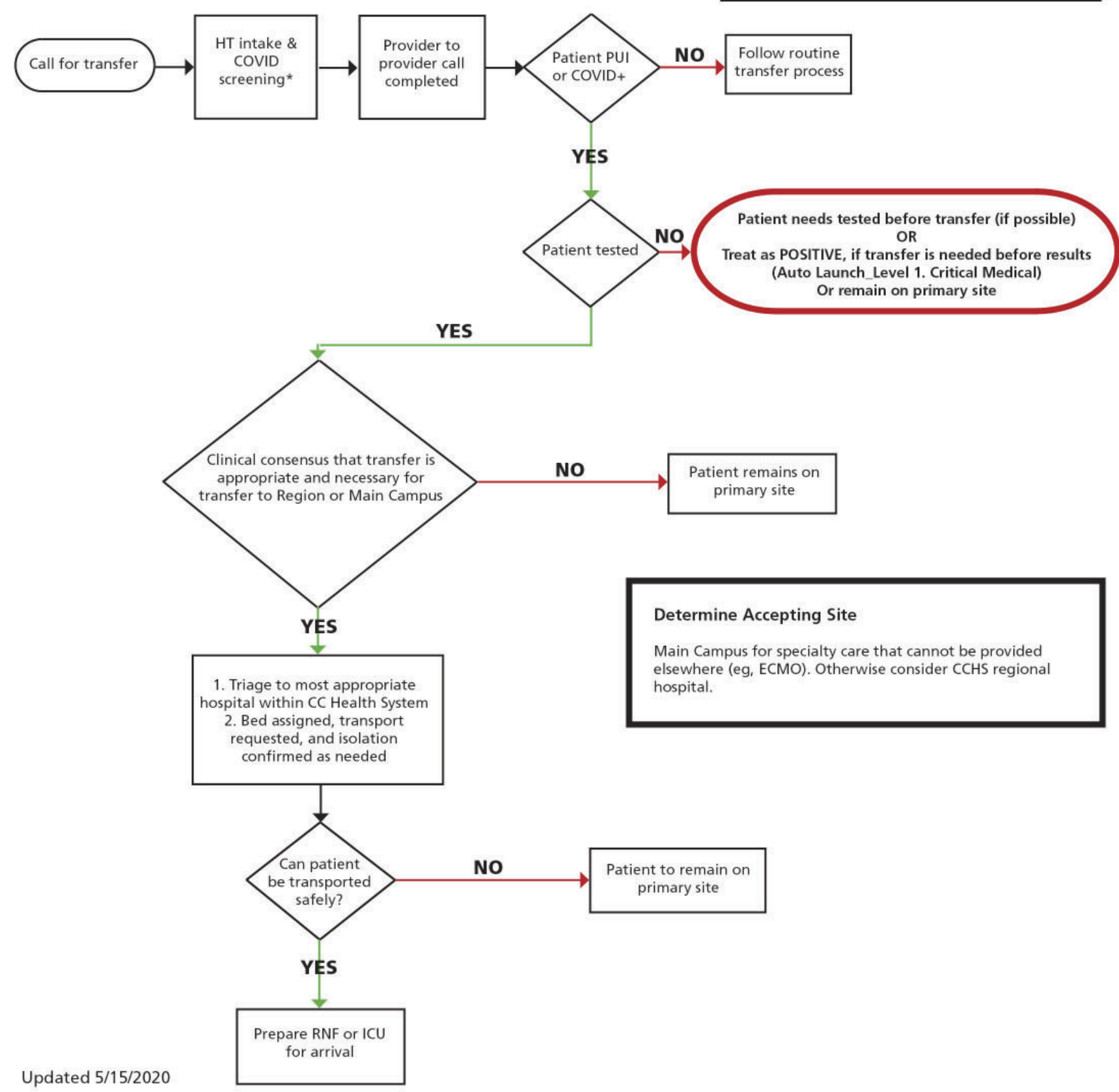

Figure 1. Cleveland Clinic algorithm for external hospital transfer of a potential or confirmed COVID-19 patient.

$\mathrm{ICU}=$ intensive care unit; $\mathrm{ECMO}$ = extracorporeal membrane oxygenation; $\mathrm{HT}$ = hospital intake; $\mathrm{PUI}=$ patient under investigation; $\mathrm{RNF}=$ radiography and fluoroscopy 


\section{PPE bag}

Base__
Vehicle Number
\begin{tabular}{|l|r|r|}
\hline N95 Mask 1860s Small & 8 & \\
\hline N95 Mask 1860 Regular & 8 & \\
\hline N95 Mask 8210 Universal/Large & 8 & \\
\hline Eye Protection/Face Shield & 8 & \\
\hline Gown & 8 & \\
\hline Numberered Seal & QS & \\
\hline Orange Bags & QS & \\
\hline
\end{tabular}

*Please use your issued reusable eye protection, clean with sani-wipe or soap and water*

Figure 2. Contents of critical care transport personal protective equipment bag.

also instituted measures to ensure that all transport personnel and nonintubated patients wear surgical masks during all phases of transport.

Adjustments to the transport space and equipment storage for each mode of transport have also been necessary. One adjustment was made because anything not enclosed in a storage cabinet was considered exposed and, thus, required cleaning and decontamination after transport of a COVID-19 patient. To avoid the extra cleaning, teams make sure to only have necessary (ie, essential) items exposed. All nonessential equipment is moved to enclosed storage cabinets. For example, if the patient transports would not require a ventilator or ultrasound, we would reposition those items in a cabinet in the truck. Air transports pose a particular challenge owing to their more confined environment, and nonessential equipment is moved to enclosed compartments near the tail.

Also, even though all team members, including pilots and EMT drivers, wear appropriate PPE, additional vehicle-specific adjustments were made for ventilation and isolation from "clean" compartments.

\section{ENSURING SAFETY}

For transport of all COVID-19-positive or suspected patients, preparation and planning is of utmost importance. A team huddle, or "time out," was instituted for every COVID-19-related patient transport and completed before entering the patient room. To minimize the risk of cross-contamination, only items deemed essential for patient care are brought into the patient room; other items are maintained in an accessible location in case they are needed.

It is vital to anticipate the need for advanced airway to decrease risk of aerosolization and potential contamination of equipment and crew. Ideally, this is performed at the referring hospital, as intubation during transport also risks contamination of pilots or EMT drivers given the confined area for procedures. For patients who are already intubated, our practice is to clamp all endotracheal tubes when transitioning from one ventilator to another to reduce aerosolization risk. Also, we added a viral bacterial filter to our equipment, placing it between the end of the ventilator circuit and the patient.

In the early stages of the pandemic and concurrent with Cleveland Clinic policy, CCT initially suspended use of high-flow nasal cannula and bilevel positive airway pressure (BiPAP) machines for transport of any suspected or confirmed COVID-19 cases owing to the aerosolization risk. However, as the situation evolved, it became apparent that this risk could be minimized by maintaining a flow rate of no more than 30 liters per minute while using a surgical mask to cover the patient's face and nose. Nevertheless, BiPAP therapy is contraindicated because of the contamination risk and is not currently used for any suspected for confirmed COVID-19 cases during transport. Finally, once the transport is underway, updates in medical condition and estimated time of arrival are relayed to the referring facility to allow time for the receiving team to prepare for transition of care and minimize the transmission risk.

\section{DECONTAMINATING TRANSPORT EQUIPMENT}

Decontamination continues to be a key component to any post-transport process for patients with confirmed or suspected COVID-19 disease. Procedures can be quickly modified to ensure a level of decontamination concordant with the COVID-19 risk. All transport surfaces potentially in contact with the patient or materials contaminated during patient care (eg, stretcher, rails, control panels, floors, walls, work surfaces) are cleaned with a bleach-based disinfection and sanitizing system (Clorox Total 360 System). Alternatively, CDC-approved decontamination wipes can be used to clean air transports to avoid spraying avionic controls. This enables all teams to safely and quickly clean all transport surfaces and minimize out-of-service time.

\section{CONCLUSION}

Overall, preparation and frequent open communication among members of the CCT during this unprecedented COVID-19 pandemic has allowed for quick adaptation of new policies and procedures, which subsequently has enabled the CCT team to continue to provide timely and high-quality patient-centered care. 


\section{REFERENCES}

1. Javed MA; Society of Critical Care Medicine. Proper transport of patients with COVID-19 from hospital to hospital. https://www. sccm.org/COVID19RapidResources/Resources/Proper-Transport-ofPatients-With-COVID-19-From-Ho. Accessed May 28, 2020.

2. West Virginia University Hospitals. Transporting of patient under COVID-19 precautions. https://wvumedicine.org/criticalcare/ wp-content/uploads/sites/31/2020/03/Policy-TRANSPORTING-OFPATIENT-UNDER-COVID-19-PRECAUTIONS.pdf. Accessed May 28, 2020.
3. Centers for Disease Control and Prevention. Coronavirus disease 2019 (COVID-19): Interim guidance for emergency medical services (EMS) systems and 911 public safety answering points (PSAPs) for COVID19 in the United States. https://www.cdc.gov/coronavirus/2019-ncov/ hcp/guidance-for-ems.html. Accessed May 28, 2020.

4. Martin T. Updated: patient air transport during the Covid-19 pandemic. AirMed\&Rescue. https://www.airmedandrescue.com/latest/ long-read/updated-patient-air-transport-during-covid-19-pandemic. Accessed May 28, 2020.

5. Liew MF, Siow WT, Yau YW, See KC. Safe patient transport for COVID-19. Crit Care 2020; 24(1):94. doi:10.1186/s13054-020-2828-4 